\title{
Histopathological study on cervical liquid-based cytological examination of ASCUS
}

\author{
Lei Yang* \\ Department of Pathology, Baogang Hospital of Inner Mongolia, Baotou, Inner Mongolia, China
}

Received: September 30, 2016

DOI: $10.14725 /$ dcc.v3n4p6
Accepted: October 25, $2016 \quad$ Online Published: December 10, 2016

URL: http://dx.doi.org/10.14725/dcc.v3n4p6

\begin{abstract}
Objective: To explore the results of cervical liquid base for atypical squamous cell histopathology.

Methods: A total of 120 cases of patients with atypical squamous cells from July 2015 to July 2016 were selected as the subjects of the study. HPV-DNA testing was intensified in patients with cervical liquid-based cytology.

Results: The diagnostic accuracy of CIN was 53.00\% (53 cases) in HPV-DNA positive group and $25.00 \%$ (5 cases) in HPVDNA negative group, and the difference was statistically significant in HPV-DNA negative group $(p<.05)$.

Conclusions: In patients with atypical squamous cell cervical liquid-based cytology test, to strengthen the detection of HPVDNA can increase the detection rate.
\end{abstract}

Key Words: The cervical liquid based cells, ASCUS, The pathological

There is no specific manifestation in the early stage of cervical lesions, whose main clinical diagnosis replies on the pathological examination of the cervical living tissue, the colposcopy and the smear of the cervix cells. The above three diagnostic methods are all effective, but there are still certain differential in the diagnosis of atypical squamous cell (ASCUS). ${ }^{[1]}$ The paper aims to explore the results of cervical liquid base for atypical squamous cell histopathology, and the concrete contents are described as follows.

\section{Method and materials}

\subsection{General information}

A total of 120 cases of patients with atypical squamous cells from July 2015 to July 2016 were selected as the subjects of the study. They are aging 22-54 years old, with an average age of $(36.89 \pm 3.48)$ years, and all the patients in the study are women. All the study subjects should meet the following requirement. (1) All patients were diagnosed with atypical squamous cell carcinoma by cervix biopsy. (2) All the patients were informed of the purpose of the study and signed the written consent form. Exclusion criteria: (1) to exclude patients with communication disorders; (2) to exclude patients with severe heart failure; (3) to exclude patients with congenital cervical deformity; (4) to exclude critical patients.

\subsection{Method}

All patients with atypical squamous cells were diagnosed with TLC, and the standard of atypical squamous cell diagnosis includes the following conditions: (1) The nuclei were slightly polluted and the outline was smooth and regular. However, some of the patients showed irregular contour, while the chromatin distribution was more uniform. (2) The cell shape and nucleus were not uniform, and even some patients had dual nucleus cells. (3) The nucleus increased

\footnotetext{
*Correspondence: Lei Yang; E-mail: dcc62@ncspress.com; Address: Department of Pathology, Baogang Hospital of Inner Mongolia, Baotou, Inner Mongolia, China.
} 
obviously. Compared with the normal nucleus, the shape of nucleus of the middle layer increased by 2.5 to 3 times. The detection kit for high risk human papillomavirus was used in this experiment. 14 kinds of high risk types of HPV-DNA were detected by Invader Technology. Positive standard: the testing results $\geq 5,000$ copies.

\subsection{Obvervational index}

The diagnostic accuracy of cervical fluid based cells in patients with atypical squamous cells was analyzed.

\subsection{Statistical method}

SPSS 21.0 statistical software was used to deal with the count data and the rate was expressed by the $\chi^{2}$ test. The difference was statistically significant with $p<.05$.

\section{Results}

Of all the 120 patients with atypical squamous cells, the high risk HPV-DNA negative rate was $16.67 \%$ (20 cases), and the positive rate was $83.33 \%$ (100 cases). After histopathological examination, 12 cases of CIN III, 12 cases of CIN II, 1 cases of cervical cancer, 19 cases of condyloma, 42 cases of cervicitis and 34 cases of CIN I were found. In the HPVDNA positive patients, 27 cases (27/100) for patients with inflammation, 19 cases (19/100) for condyloma patients, 30 cases (30/100) for CIN I patients, 11 cases (11/100) for CIN II patients, 12 cases (12/100) for CIN III patients, 1 cases (1/100) for cervical cancer patients. In patients with HPVDNA negative group, 15 cases (15/20) were inflammatory patients, 4 cases (4/20) were CIN I patients, and 1 case $(1 / 20)$ was CIN II patients. The diagnostic accuracy of CIN in HPV-DNA positive group was 53\% (53/100), while in HPV negative group, the diagnostic accuracy of CIN was $25 \%(5 / 20)$. The difference between the two groups was statistically significant $(p<.05)$.

\section{Discussion}

At present, the clinical test technique is difficult to distinguish the patients with atypical squamous cells. It may be tracked to the following causes if there are obvious changes in atypical squamous cells: (1) cervical intraepithelial neoplasia; (2) the influence of intrauterine device; (3) inflammation. $^{[2,3]}$ Atypical squamous cell cytology is mainly divided into two types of lesions, malignant or benign. We cannot make an accurate diagnosis of the patient's condition by pathological examination, which indicates the seriousness of the disease only. Clinical studies have confirmed that atypical squamous cells appear to be at risk of cervical intraepithelial neoplasia when cytological changes occur. Some patients can develop canceration phenomenon. ${ }^{[4]}$ Therefore, there are two possible results for the diagnosis of atypical squamous cells. One of which is an over-diagnosis of reactive cytological changes, the other is to underestimate the incidence of precancerous lesions of the cervix. In this study, the results of atypical squamous cell pathology were significantly different. ${ }^{[5]}$

To date, the following methods are commonly used in the diagnosis of atypical squamous cells. (1) Colposcopy examination was performed for patients and guidance of pathological biopsy results was provided; (2) High-risk HPV-DNA detection was carried out for patients; (3) Cytological examination was repeated for many times, and follow-up was strengthened. ${ }^{[6]}$ Repeated cytological examination is not widely used clinically since it may cause greater psychological burden and economic pressure for the patients. However, it not only can improve the accuracy of clinical diagnosis during the process of high-risk HPV-DNA detection but also reflect the cytological changes of patients, timely detection of potential cancer patients, is conducive to the clinical treatment. ${ }^{[7,8]}$

In 2006, the study of atypical squamous cell patients was conducted by the foreign society of cervical pathology and the pathological association of colposcopy. It was reported that 2 times of colposcopy, high risk HPV-DNA, and cytological examination were carried out for patients at intervals of 6 months. The results showed that the cytological examination could eliminate the false negative results of the patients, and could effectively prevent the false negative pathology from the missed diagnosis. Therefore, for the patients with cervical lesions, the primary treatment principle is early detection, early diagnosis and early intervention, which is conducive to the recovery of the patient's condition. Through this study, we found that the diagnostic accuracy of CIN in HPV-DNA positive group was 53\% (53 cases), while in HPV-DNA negative group, the diagnostic accuracy of CIN was $25 \%$ (5 cases). It shows that cervical liquid based cell examination has certain diagnostic value for cervical patients, which not only can make up for the shortcomings of early diagnosis methods, but also timely distinguish the location and nature of cervical lesions, so as to provide an important basis for clinical treatment.

To sum up, in the implementation of cervical liquid based cell examination for patients with atypical squamous cell carcinoma, strengthening the detection of HPV-DNA can reduce the clinical misdiagnosis rate, and provide important basis for clinical treatment.

\section{Conflicts of Interest Disclosure}

The authors have no conflicts of interest related to this article. 


\section{References}

[1] Chen XH. The diagnostic value of cervical fluid based cytology in cervical lesions. Maternal and Child Health Care of China. 2012; 27(18): 2890-2891.

[2] Yang LL, Yang HL, Du XM, et al. Clinical treatment of atypical squamous epithelial cells without definite diagnostic significance. Journal of Practical Obstetrics and Gynecology. 2011; 27(18): 3133.

[3] Du R, Chen ZF, Ding Y. The value of high risk human papillomavirus detection in atypical squamous epithelial cells of undefined diagnostic significance of the cervix. Journal of Practical Obstetrics and Gynecology. 2011; 27(8): 603-606.

[4] Wang ZM, Chen Y, Zheng JP. The diagnostic validity of liquid based cytology combined with high risk HPV-DNA for precancer- ous lesions of the cervix. Chinese Journal of Pathophysiology. 2011; 27(12): 2414-2416

[5] Guo RL. Study on the application value of cervical liquid based cell screening in screening of cervical cancer. Journal of Practical Gynecologic Endocrinology. 2015; 2(11): 113-114.

[6] Mo XL, Wei RG, Lu JY, et al. The value of Sirt1 and ProExC in cytological diagnosis of high squamous intraepithelial lesions of the cervix. Chinese Journal of Laboratory Diagnosis. 2015; 19(11): 1886-1889.

[7] $\mathrm{Xu} \mathrm{H}$, Yao MX, Huang N, et al. The value of high risk human papillomavirus infection detection in the management of cervical atypical squamous epithelial cells in women. Chinese Journal of Nosocomiology. 2014; 24(4): 809-810, 813.

[8] Zhao H, Pan HY, Lu S, et al. The clinical value of liquid based cytology, biopsy under colposcopy and LEEP in the diagnosis and treatment of cervical intraepithelial neoplasia. The Journal of Practical Medicine. 2013; 29(18): 2989-2991. 